\title{
Os efeitos do NAFTA na destruição do México para justificar políticas comerciais transnacionais e alterações na cultura alimentar
}

\section{The effects of NAFTA on the destruction of Mexico to justify transnational trade policies and changes in food culture}

\section{Resenha do livro:}

GÁLVEZ, Alyshia. Eating NAFTA: Trade, Food Policies and the Destruction of Mexico. Berkeley: University of California Press, 2018.304 p.

\section{Ewerton Reubens Coelho-Costa'}

RESUMO: A obra Eating NAFTA: Trade, Food Policies and the Destruction of Mexico (2018), de Alyshia Gálvez, publicada em inglês pela editora da Universidade da Califórnia, e ainda sem tradução no Brasil, esclarece detalhadamente e de maneira oportuna a relação entre a perda da biodiversidade, a migração, as alterações na cultura alimentar mexicana, as doenças relacionadas à dieta e os acordos comerciais internacionais. Com foco no Acordo de Livre Comércio da América do Norte (Nafta), em vigor desde 1994, analisa as duas décadas seguintes ao acordo evidenciando seu impacto na vida dos mexicanos que vivem nos dois lados da fronteira. Para além da obra resenhada, este texto apresenta os últimos desdobramentos que levaram às implicações e alteração do NAFTA transformando-o em

\footnotetext{
${ }^{1}$ Doutorando em Sociologia pela Universidade Estadual do Ceará (Bolsista CAPES); Mestre em Gestão de Negócios Turísticos - UECE; Graduado em Gestão do Turismo (IFCE); Membro do Grupo de Pesquisa Gestão do Turismo e da Hospitalidade nos Territórios no Instituto Federal de Educação, Ciência e Tecnologia do Ceará IFCE (Campus Fortaleza). Dedicado a pesquisas com ênfase em Cultura Castronômica, Turismo Gastronômico e Enogastronômico, Indicações Geográficas Brasileiras, Patrimônio Gastronômico e Gastrodiplomacia. Contato: ewertonreubens@gmail.com.
} 
Acordo Estados Unidos-México-Canadá (USMCA, na sigla em inglês para United StatesMexico-Canada Agreement).

Palavras-chave: Nafta; políticas alimentares; migração; políticas transnacionais.

ABSTRACT: Eating NAFTA: Trade, Food Policies and the Destruction of Mexico (2018), by Alyshia Gálvez, published in English by the University of California Press, and still without translation in Brazil, clarifies in detail and in a timely manner the relationship between loss biodiversity, migration, changes in Mexican food culture, diet-related illnesses and international trade agreements. Focusing on the North American Free Trade Agreement (Nafta), in force since 1994, it analyzes the two decades following the agreement, showing its impact on the lives of Mexicans living on both sides of the border. In addition to the work reviewed, this text presents the latest developments that led to the implications and alteration of NAFTA, transforming it into the United States-Mexico-Canada Agreement (USMCA).

Keywords: Nafta; food policies; migration; transnational policies.

Nafta é a sigla em inglês de North American Free Trade Agreement, para o acordo econômico que se conhece no Brasil como Tratado Norte-Americano de Livre Comércio, assinado pelos representantes do poder executivo dos Estados Unidos da América, Canadá e México. Basicamente, o tratado tem como objetivo eliminar ou reduzir barreiras comerciais entre os signatários desde que foi criado, para competir com a União Europeia. Contudo, diferentemente do bloco europeu, o Nafta não permite o livre trânsito de cidadão dos países signatários nem possui moeda própria. E alguns cientistas não digerem as circunstâncias desse acordo.

Assim, a obra Eating NAFTA: Trade, Food Policies and the Destruction of Mexico (2018), de Alyshia Gálvez, publicada em inglês pela editora da Universidade da Califórnia, ainda sem edição em português, esclarece detalhadamente esse panorama.

Alyshia Gálvez é antropóloga cultural, com especialização na área da alimentação, saúde, política, migração e direitos dos cidadãos latinos, professora de estudos latino- 
americanos e latinos no Lehman College da City University of New York (CUNY). Foi diretorafundadora do Instituto de Estudos Mexicanos Jaime Lucero, na CUNY, e autora de dois livros anteriores sobre migração mexicana: Patient Citizens, Immigrant Mothers: Mexican Women, Public Prenatal Care, and the Birth Weight Paradox (2011), livro vencedor do prêmio ALLA Book Award 2012 da Associação de Antropólogos Latinos e Latino-Americanos, e Guadalupe in New York: Devotion and the Struggle for Citizenship Rights Among Mexican Immigrants (2010). A autora dedica suas pesquisas a estudos latinos e latino-americanos geralmente analisando mudanças políticas, sistemas e práticas alimentares, política comercial e econômica, e suas implicações na saúde pública.

Alyshia Gálvez elaborou um livro oportuno para discutir a relação entre a perda da biodiversidade, a migração, as doenças relacionadas à dieta e os acordos comerciais internacionais. Com foco no Nafta, em vigor desde 1994, analisa as duas décadas seguintes ao acordo evidenciando seu impacto na vida dos mexicanos que vivem nos dois lados da fronteira.

A obra está dividida em oito capítulos que se distribuem ao longo de 288 páginas. Com estatísticas de fontes confiáveis, Gálvez observa os dados e os junta a seus encontros etnográficos com pessoas de várias classes sociais, urbanas e rurais, políticos, trabalhadores e migrantes. A autora rende atenção especial à mudança do estilo de vida rural de mexicanos que deixaram de poder sustentar sua agricultura milpa, a partir do momento em que os Estados Unidos subsidiam milho às toneladas para plantio no México. Isso não mudou apenas as dietas, mas os impediu de manter domicílio geracional, já que membros da família acabaram migrando para fora do país na busca de dinheiro.

Alyshia Gálvez revela que, atualmente, o México tem uma taxa de pobreza de 55,1\%; importa $42 \%$ de seus alimentos; e tem doenças crônicas ligadas à dieta como as três principais causas de incapacidade e morte. Além disso, mostra que 12,8 milhões de mexicanos residiam nos Estados Unidos no ano de 2007. Assim, desde a introdução (p. 1-26)², a autora encara esses fatos como um tipo de violência estrutural: "gringos clamam por tortillas feitas à mão, enquanto os mexicanos se tornaram os maiores consumidores de macarrão instantâneo do mundo" (p. 10).

\footnotetext{
${ }^{2}$ Doravante, as citações remetem à obra resenhada (CÁLVEZ, 2018) e foram vertidas, livremente, para o português.
} 
No segundo capítulo, People of the Corn (p. 27-62), Gálvez apresenta uma visão etnográfica de como a comida mexicana tradicional entrou para o mundo da haute cuisine, a partir das comparações elegidas pelo renomado chef dinamarquês René Redzepi, dono do premiado restaurante Noma. A autora analisa o papel do capital narrativo em repassar histórias romantizadas de elementos específicos da culinária, como o milho, enquanto informa sobre condições históricas de produção, desenvolvimento e custódia desses elementos ao longo de milênios, até chegar à inclusão da cozinha mexicana na lista de Patrimônio Cultural Imaterial da Unesco, em 2010.

A autora ainda aborda essa entrada na lista da Unesco ao mesmo tempo que fornece exemplos das mudanças na cozinha mexicana sofridas desde o período de conquista pelos espanhóis até o Nafta. Apresenta o caso do milho landrace (termo geral e impreciso para 59 variedades de milhos nativos mexicanos adaptados localmente, cultivados e consumidos por proprietários de terras tradicionais naquele país - não se trata do milho amarelo-dourado e do milho doce convencional, ambos de uma ampla população de variedades com formas, cores, texturas e sabores diferentes), antes utilizado por fazendeiros para alimentar seus animais, uma vez que a demanda por ele e seus preços eram baixos. Hoje, no entanto, esse tipo de milho foi descoberto pelas elites e por chefs de cozinha que pagam preços altos por ele e o servem em odes gastronômicas com outros elementos tradicionais da cozinha mexicana, como mezcal, huitlacoche (espiga de milho contaminada pelo fungo Ustilago maydis, uma praga que ataca milharais pelo mundo) e até larvas de insetos.

No capítulo terceiro, Laying the Groundwork for NAFTA (p. 63-88), tirando o foco do milho e levando para a criação de cabras crioulas, a autora apresenta o panorama do desejo mexicano de tornar sua produção agrícola mais eficiente. Para tanto, ensaia a história de desenvolvimento do México, revelando que a agricultura de pequena escala garante a biodiversidade e a sustentabilidade ambiental e é compatível com outras atividades econômicas e de subsistência; ao mesmo tempo, revela o contraste de um modelo de segurança alimentar regido por acordos de livre comércio, como o Nafta, e um modelo de soberania alimentar que exige controle democrático do sistema alimentar - aqui se explica, por exemplo, como produtores rurais são marginalizados e tidos como culpados pela ineficiência do México e como os deslocamentos aumentaram a migração e o consumo de produtos dos Estados Unidos, contribuindo com o crescimento da economia dos Estados Unidos 
O quarto capítulo, Nafta: Free Trade in the Body (p. 89-116), inicia-se com a descrição da loja de doces e alimentos processados de Doña Yolanda e o esclarecimento de que a atração por esse tipo de negócio, principalmente em países pobres, se desenvolve, sobretudo, como forma de enfrentar a competição com grandes redes como Walmart e Oxxo. Fica evidente a falsa ideia de modernidade e cosmopolitismo trazida com esses alimentos processados e a transformação deles, na atualidade, em produtos considerados de baixo status, ressaltando que o livre comércio ocasionou também uma transição nutricional marcada pela obesidade generalizada, devido à maior quantidade de açúcares e elementos químicos usados na produção desses alimentos. Fato assustador é constatar que, com a aprovação do Nafta, as exportações de produtos químicos dos Estados Unidos para o México aumentarem em 97\% (uma realidade semelhante ao que vem ocorrendo no Brasil desde que o governo Bolsonaro subiu ao poder).

O quinto capítulo, Deflecting the Blame: Poverty and Personal Responsibility (p. 117-158), aborda o combate ao diabetes e à obesidade no México, refletindo que o problema parece recair apenas sobre o indivíduo e não nas mudanças do sistema alimentar. Gálvez descreve três partes da resposta do governo mexicano ao diabetes e à obesidade: o imposto sobre o refrigerante e os regulamentos sobre marketing de alimentos; o programa Anti-Pobreza que prospera junto da Cruzada Contra a Fome; e como as duas últimas estratégias impulsionam as pessoas para uma economia baseada em dinheiro, longe do conhecimento tradicional sobre alimentos saudáveis. Esse capítulo finaliza discutindo o trabalho das mulheres e como, mesmo quando elas trabalham fora de casa, espera-se que sejam as únicas responsáveis pelas dietas de suas famílias; e justifica que, à medida que o acesso ao dinheiro aumenta e o tempo diminui, é mais provável que elas confiem em alimentos processados. A autora ressalta que não é justo que o Estado não seja responsabilizado por esses problemas, sobretudo, quando é ele quem assina acordos e que, portanto, deveria pensar em seus impactos.

O sexto capítulo, Diabetes: The Disease of the Migrant? (p. 159-172), lança olhar sobre o papel da migração no surgimento e no aumento de diabetes no México e foca nas relações entre estresse e essa doença versus migração e estresse. Com reflexos dos estudos de Mendenhall (2017) sobre o sofrimento sindêmico, a autora chama a atenção para a interseção de ambas as doenças e os problemas sociais causados por epidemia. Cabe mencionar a relevância do uso da "sindemia" na análise do texto. A sindemia, conceito recente criado por 
Merril Singer, trata do agrupamento de dois ou mais problemas de saúde de uma população, que resulta em desigualdades sociais e econômicas persistentes; considera os aspectos biológicos, psicológicos e sociais nas análises, embora sejam enfermidades crônicas não transmissíveis (SINGER, 2009; 2000).

Ainda ficam evidentes no sexto capítulo as conexões entre os tipos de estresse e o diabetes, ressaltando situações corriqueiras como a separação dos membros da família, a discriminação, a exploração do trabalho, a pobreza e a falta de seguro de saúde como elementos que acabam mudando as dietas dos migrantes - principalmente quando eles resolvem voltar para sua pátria, mas mantêm o consumo de grande quantidade de alimentos processados.

O sétimo capítulo, Nostalgia, Prestige, and a Party Every Day (p. 173-188), apresenta o agrião, um alimento que muitos latino-americanos utilizam, mas que não é visto nos estereótipos da comida latina, o que leva Gálvez a interrogar: "quantos alimentos humildes, mas claramente significativos, são esquecidos na transição para estilos de vida mais urbanos ou com a migração?" (p. 174). A autora ainda argumenta sobre o quanto nossa nostalgia alimentar pode ser feita de contextos produzidos e como a migração intensifica essa nostalgia por gostos e lugares específicos, principalmente em ambientes onde a livre circulação é impedida. Pelo fato da descontextualização ocorrem apropriações e a mercantilização de alimentos tradicionais - como os citados casos dos tamales oferecidos pela Williams-Sonoma; e o McBurrito, do McDonald's. Como enfrentamento a essa realidade, surgem os ativistas alimentares que promovem alimentos tradicionais, explorando seus benefícios para a saúde e os tratando como parte da soberania alimentar. Enquanto a culinária tradicional mexicana está se tornando global, bebidas como a Coca-Cola constituem elementos globais que invadem as culturas, e os rituais indígenas acabam se tornando mais uma ferramenta útil de marketing.

Na conclusão, Connecting the Dots, and Bright Spots (p. 189-202), surge a história de um migrante mexicano em Nova York, que faz o caminho de volta à sua terra com a ajuda de uma organização sem fins lucrativos chamada GrowNYC. Discute-se com isso o contrate com a multidão de migrantes mexicanos que se tornaram "corpos excedentes" e "corpos como repositórios de excedentes, armazenando os produtos de superprodução e negociações comerciais desiguais" (p. 192). Gálvez constata que o aumento das doenças relacionadas com a dieta no México é "resultado lógico da priorização do investimento estrangeiro direto, da 
agricultura industrial, das teorias de vantagem comparativa e de um papel específico de desenvolvimento que não vê nenhum papel para a agricultura de pequena escala" (p. 192193).

Gálvez leva os leitores a conhecer movimentos alternativos, como o referido GrowNYC, que promovem a justiça social, a resistência e a resiliência enquanto promovem maneiras de comer repletas de simbologias e conexões entre si, a terra e a cultura. Mas ela adverte que as soluções para os problemas analisados exigem mais do que ativismo do consumidor em nível local.

Mudando o discurso, muitas vezes, ao longo do livro, Gálvez oferece ao leitor uma visão sobre as conversas e observações que a levaram a criar a obra: às vezes, são descrições de encontros; outras vezes, transcrições diretas de entrevistas em espanhol. Estas não são traduzidas no texto, mas apenas resumidas e comentadas. Os leitores mais interessados podem encontrar as traduções exatas nas notas finais.

A escrita de Gálvez tem um estilo refrescante que mantém o interesse do leitor nos tópicos em questão e, ao mesmo tempo, abre a cortina de pesquisa. Fica claro o sucesso de Gálvez em apresentar a complexidade de um sistema alimentar que deu errado e o importante papel desempenhado pelo Nafta. Trata-se de um texto altamente recomendado para curiosos e pesquisadores que lidam com sistemas alimentares, justiça social, migração, políticas públicas e ciências sociais - bem como para cursos mais específicos que têm foco sobre a América Latina e no México.

No entanto, enquanto resenhava a obra, o mundo dos acordos políticos seguia a todo vapor, a ponto de levar os Estados Unidos e o Canadá a um acordo para substituir o $\mathrm{Nafta}^{3}$. Assim, surge o novo tratado, com o nome de Acordo Estados Unidos-México-Canadá (USMCA, na sigla em inglês para United States-Mexico-Canada Agreement), e que representa uma vitória do presidente Donald Trump, que considerava o Nafta um "desastre" para os Estados Unidos ${ }^{4}$.

A assinatura do novo acordo resgata uma zona de livre comércio entre os três países, equivalente a 1,2 trilhão de dólares por ano em comércio, que estava prestes a entrar em

\footnotetext{
${ }^{3}$ Cf. CANADÁ e EUA chegam a acordo para substituir o Nafta a poucas horas do prazo limite. G1 Economia, São Paulo, 01 out. 2018. Disponível em: <https://g1.globo.com/economia/noticia/2018/10/01/canada-e-euachegam-a-acordo-para-substituir-o-nafta-a-poucas-horas-do-prazo-limite.ghtml>. Acesso em: 04 nov. 2018.

${ }^{4}$ Cf. UNITED STATES-MEXICO-CANADA AGREEMENT. Office of the United States Trade Representative. Washington, 2018. Disponível em: <https://ustr.gov/trade-agreements/free-trade-agreements/united-statesmexico-canada-agreement>. Acesso em: 11 ago. 2020.
} 
colapso após quase 25 anos. As renegociações iniciaram-se em 2017, quando México e Estados Unidos já haviam fechado um acordo preliminar, mas faltava o Canadá. Depois de mais de um ano em negociações para superar divergências e ceder em alguns aspectos, os representantes das três nações comemoram o USMCA, que movimentará e influenciará uma área de 500 milhões de pessoas.

Partindo das notícias veiculadas nas mais diversas mídias, o novo acordo empolgou os mercados globais. No entanto, começa agora uma nova possibilidade de pesquisa para Gálvez, ou mesmo para qualquer um de nós, para (re)avaliar os impactos desse novo acordo na alimentação e na saúde dos países signatários.

\section{Referências}

CÁLVEZ, Alyshia. Guadalupe in New York: Devotion and the Struggle for Citizenship Rights Among Mexican Immigrants. Nova York/Londres: New York University Press, 2010.

GÁLVEZ, Alyshia. Patient Citizens, Immigrant Mothers: Mexican Women, Public Prenatal Care, and the Birth Weight Paradox. New Brunswick: Rutgers University Press, 2011.

GÁLVEZ, Alyshia. Eating NAFTA: Trade, Food Policies and the Destruction of Mexico. Berkeley: University of California Press, 2018.

MENDENHALL, Emilly. Syndemics: A New Path for Global Health Research. The Lancet, v. 389, n. 10072, p. 889-891, 2017.

SINCER, Merrill. A Dose of Drugs, a Touch of Violence, a Case of Aids: Conceptualizing the Sava Syndemic. Free Inquiry in Creative Sociology, v. 28, n. 1, p. 13-24, 2000.

SINGER, Merrill. Introduction to Syndemics: A Critical Systems Approach to Public and Community Health. San Francisco: Jossey-Bass, 2009. 